\title{
Radiative properties of an electrically-vaporized thin film plasma in an external magnetic field
}

\author{
D. Albers and R. SACKS* \\ Department of Chemistry, University of Michigan, Ann Arbor, MI 48109, U.S.A.
}

(Received 12 September 1985)

\begin{abstract}
An external magnetic field of a few kilogauss is used to alter significantly the radiative properties of the plasmas generated by the capacitive discharge vaporization of thin $\mathrm{Ag}$ films. The magnetic field is generated by the plasma current in a large inductor surrounding the plasma. The magnetic field is oriented normal to the electric field in the plasma. This generates a drift motion of the plasma normal to the plane established by the electric and magnetic field vectors. The drift motion can direct the plasma toward the plastic substrate on which the $\mathrm{Ag}$ film and a powder or solution residue sample were originally located. This increases the plasma-sample interaction. Time integrated, spatially resolved photographic spectra and spatially resolved photoelectric radiation profiles show that with the magnetic field, the continuum background radiation is confined to a relatively small region near the substrate surface. This results in significantly larger analyte line-to-background intensity ratios in the region $1-2 \mathrm{~cm}$ above the substrate surface.
\end{abstract}

\section{INTRODUCTION}

THE USE of externally applied magnetic fields for the control of high-temperature plasmas for analytically atomic spectroscopy represents a relatively unexplored area. Relatively modest magnetic fields of a few kilogauss can alter significantly the macroscopic properties of the plasma as well as the motions of individual ions and electrons. While external magnetic fields have been used in conjunction with d.c. arcs [1-3], high-voltage sparks [4], microwave induced plasmas [5] and glow discharge lamps $[6,7]$, there have been relatively few definitive reports on the interactions of magnetic fields with analytically useful plasmas. Recently, ALBERs et al. [8] discussed several magnetic field-plasma configurations in which the transient plasma current from a capacitive discharge was used to generate the magnetic field in a coil surrounding the plasma.

If a macroscopic electric field is present in the plasma, an external magnetic field with a component normal to the electric field will generate a drift motion in the plasma which is normal to the plane containing the electric and magnetic field vectors. This motion is the result of coulombic perturbation of the Larmor orbits of ions and electrons as they precess in the plane normal to the magnetic field. Often this is referred to as an $E \times B$ drift motion $[9,10]$. The drift velocity $v_{d}$ is given by Eqn (1).

$$
v_{\mathrm{d}}=\bar{E} \times \bar{B} / B^{2}
$$

where $\bar{E}$ and $\bar{B}$ are the electric and magnetic field vectors, respectively, and $B$ is the scalar magnetic field strength. If the plasma is highly ionized, this drift motion can be a dominant factor in determining the properties of the plasma. Preliminary studies [8] with an electrically

*Author to whom correspondence should be directed.

[1] V. Vukanović, V. Georguivić, D. Vukanović and M. Todorović, Spectrochim. Acta 24B, 555 (1969).

[2] M. Todorovic, V. Vukanović and V. GeorgiJević, Spectrochim. Acta 24B, 571 (1969).

[3] A. A. FAKHRY, M. A. EID and M. S. HASHEM, Spectrochim. Acta 32B, 272 (1978).

[4] R. J. KLUEPPEL and J. P. WALTERS, Spectrochim. Acta 35B, 431 (1980).

[5] S. R. GOODE and D. T. PIPES, Spectrochim. Acta 36B, 925 (1981).

[6] R. A. Kruger, R. M. BombelKA and K. LAQUA, Spectrochim. Acta 35B, 581 (1980).

[7] J. A. THORNTON, J. Vac. Sci. Tech. 16, 79 (1979).

[8] D. Albers, E. Johnson, M. TisACK and R. SACKS, Appl. Spectrosc. 40, 000 (1986).

[9] F. F. CHEN, Introduction to Plasma Physics. Plenum Press, New York (1974).

[10] T. J. M. Boyd and J. J. Sanderson, Plasma Dynamics. Barnes \& Noble, New York (1969). 
vaporized thin film plasma [11-14] have shown that an external magnetic field of a few kilogauss normal to the electric field in the plasma can dramatically change the size, shape and location of the plasma current channel. If the $E \times B$ motion of the plasma is directed toward the surface of the plastic substrate which serves as a support for the thin metal film, the plasma is kept in contact with the substrate for the entire duration of the discharge. This results in more complete vaporization of the thin film and presumably more efficient interaction between the plasma and a powder or solution-residue sample initially placed on the film surface.

If the connections between the thin film and the rest of the discharge circuit are reversed thus reversing the direction of the electric field in the plasma, the $E \times B$ drift motion is directed away from the substrate surface; the plasma rapidly leaves the observation zone, and most of the thin film and presumably most of the sample are left unvaporized at the end of the discharge.

In the study reported here, time integrated, spatially resolved photographic spectra and spatially resolved photoelectric radiation profiles are used to obtain more definitive information on the $E \times B$ thin film plasma. Plasma expansion velocity and time and spatially resolved analyte line and continuum background intensities and line-to-background intensity ratios are compared with and without an external magnetic field for two values of discharge circuit inductance and voltage.

\section{Apparatus and Experiment Design}

\subsection{Magnetic field generation and plasma-field orientation}

The high-current capacitive discharge circuit used to generate the plasma also was used to generate the external magnetic field. Large air-core inductors have been used in previous electrically vaporized thin film studies to decrease ringing frequency and increase plasma duration $[12,14]$. In the present study, a $105 \mu \mathrm{H}$ inductor was redesigned to fit on the nylon discharge chamber. This inductor was in series with the rest of the discharge circuit. The inductor is $12.5 \mathrm{~cm}$ long and $19 \mathrm{~cm}$ in diameter and consists of 25 turns of AWG-14 solid copper wire molded in an epoxy resin. The inductor was designed to be easily removed from the chamber so that the plasma properties could be compared with and without the external field while always keeping the inductor in the discharge circuit.

Figure 1 shows the inductor orientation with respect to the thin $\mathbf{A g}$ film. The inductor axis and thus the magnetic field axis is parallel to the optical axis but normal to the electric field axis in the plasma. The $E \times B$ drift motion of the plasma then can be changed to vertically up or down simply by reversing the connecting leads between the thin film and the rest of the discharge circuit.

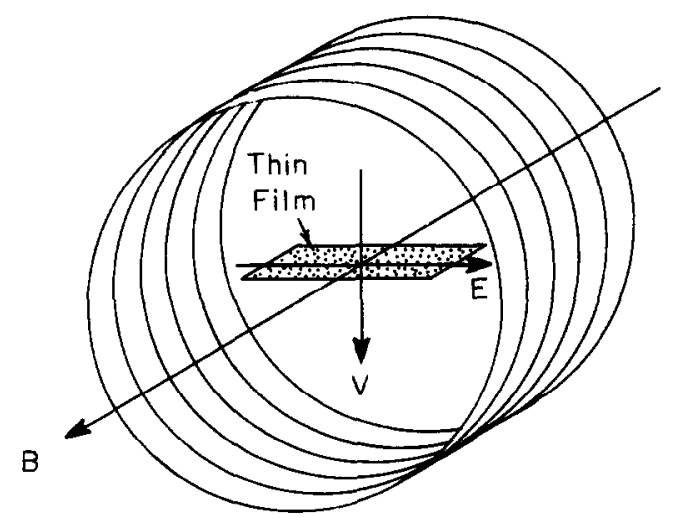

Fig. 1. Orientation of the external magnetic field coil with respect to the thin $\mathrm{Ag}$ film. The $E \times B$ drift motion of the plasma is parallel to the vertical axis. The direction of the drift motion (up or down) can be changed by reversing the connections between the coil and the rest of the discharge circuit.

[11] S. Y. Suh, R. J. Collins and R. D. SACKS, Appl. Spectrosc. 35, 42 (1981).

[12] J. Goldberg and R. SACKS, Anal. Chem. 54, 2179 (1982).

[13] R. D. Sacks, J. M. Goldberg, R. J. Collins and S. Y. Suh, Prog. Analyt. Atom. Spectrosc. 5, 111 (1982).

[14] J. Swan and R. SACKS, Anal. Chem. 57, (1985). 


\subsection{Capacitive discharge circuit and associated apparatus}

The discharge circuit is similar to previous designs [11]. A $30 \mu \mathrm{F}$ capacitor bank is charged to either 6.0 or $8.0 \mathrm{kV}$ and discharged on command through a loop containing the thin $\mathrm{Ag}$ film, the fieldproducing inductor and a high-current switch. The switches are described elsewhere [11]. In some cases, an additional $835 \mu \mathrm{H}$ of inductance was added to the discharge circuit. Table 1 lists the discharge conditions and the thin film and substrate properties.

All experiments were conducted in a $18 \mathrm{~cm}$ diameter nylon chamber. The chamber and the cassette used to support the thin film on its plastic substrate are similar to previous designs $[15,16]$ except the chamber axis was oriented parallel to the optical axis to accomodate the inductor assembly. Electrical contact to the thin film was provided by graphite electrodes in the cassette. Radiation was viewed through quartz windows mounted in the ends of the chamber.

\subsection{Radiation and electrical measurements}

All spectra were obtained with a $0.75 \mathrm{~m}$ Czerny-Turner plane grating spectrometer which was used in the first order with a 1200 line/mm grating. Reciprocal linear dispersion was about $1.05 \mathrm{~nm} / \mathrm{mm}$. Photoelectric measurements were made with a 1P28B photomultiplier tube using a $1 \mathrm{k} \Omega$ resistive load in an operational amplifier current follower.

The image transfer system used for spatially resolved radiation measurements consists of two $50 \mathrm{~mm}$ diameter, $310 \mathrm{~mm}$ focal length mirrors in an over- and under-configuration as shown in Fig. 2. The tangential image of the central plane of the plasma was located in the plane of the spectrometer entrance slit. This results in spatial resolution of plasma radiation in the direction of the drift motion ( $Y$ axis), and some integration in the direction parallel to the electric field axis ( $X$ axis). Since plasma dimensions may be greater than the $20 \mathrm{~mm}$ length of the entrance slit, a magnification of about 0.3 was used in the image transfer system.

For spatially resolved photoelectric radiation measurements, a $1 \mathrm{~mm}$ long entrance slit was used so that a $3.3 \mathrm{~mm}$ high region of the plasma was observed. The observation window scaled to the dimensions of the cassette assembly is shown in the Fig. 2 inset. Note that plastic shields are used to prevent sparkover to metallic parts of the cassette. A small space is left between the bottoms of the shields and the thin film surface. The plasma must pass through these slots, and thus it is mechanically confined in these regions near the electrodes. This improves positional stability of the plasma and increases analytical reproducibility. In the absence of the external magnetic field, the plasma can expand freely in the region between the shields. The plasma chamber was translated along a vertical axis ( $Y$ axis) for viewing various regions above the substrate surface. Photoelectric radiation measurements were made with observation windows centered at $0,5,10,15$ and $20 \mathrm{~mm}$ above the substrate surface. Time

Table 1. Thin film/substrate properties and discharge conditions

\begin{tabular}{|c|c|c|}
\hline \multicolumn{3}{|l|}{ Thin film and substrate properties } \\
\hline Substrate material & \multicolumn{2}{|c|}{ Polyethylene } \\
\hline Ag film dimensions $(\mathrm{cm})$ & \multicolumn{2}{|c|}{$7.3 \times 1.6$} \\
\hline Ag film area vaporized $\left(\mathrm{cm}^{2}\right)$ & \multicolumn{2}{|c|}{7.9} \\
\hline Ag mass vaporized $(\mu \mathrm{g})$ & \multicolumn{2}{|c|}{350} \\
\hline Ag film thickness (nm) & \multicolumn{2}{|c|}{40} \\
\hline Ag film resistance ( $\Omega$ ) & \multicolumn{2}{|c|}{$3-7$} \\
\hline \multicolumn{3}{|l|}{ Coil construction } \\
\hline Material & \multicolumn{2}{|c|}{ AWG-14 solid copper } \\
\hline Dimensions (cm) & \multicolumn{2}{|c|}{25 turns, 12.5 long, 19 diameter } \\
\hline \multicolumn{3}{|l|}{ Discharge conditions } \\
\hline Charging voltage (kV) & 6.0 & 8.0 \\
\hline Inductance $(\mu \mathrm{H})$ & 105 & 940 \\
\hline Capacitance $(\mu \mathrm{f})$ & 30 & 30 \\
\hline Energy (J) & 540 & 960 \\
\hline Ringing frequency $(\mathrm{kHz})$ & 2.8 & 0.95 \\
\hline Peak current (kA) & 2.78 & 1.24 \\
\hline Peak magnetic field (kG) & 3.7 & 1.7 \\
\hline Support gas & \multicolumn{2}{|c|}{$\operatorname{Ar}(60 \%)-\mathrm{O}_{2}(40 \%)$} \\
\hline Pressure & \multicolumn{2}{|c|}{ Atmospheric } \\
\hline
\end{tabular}




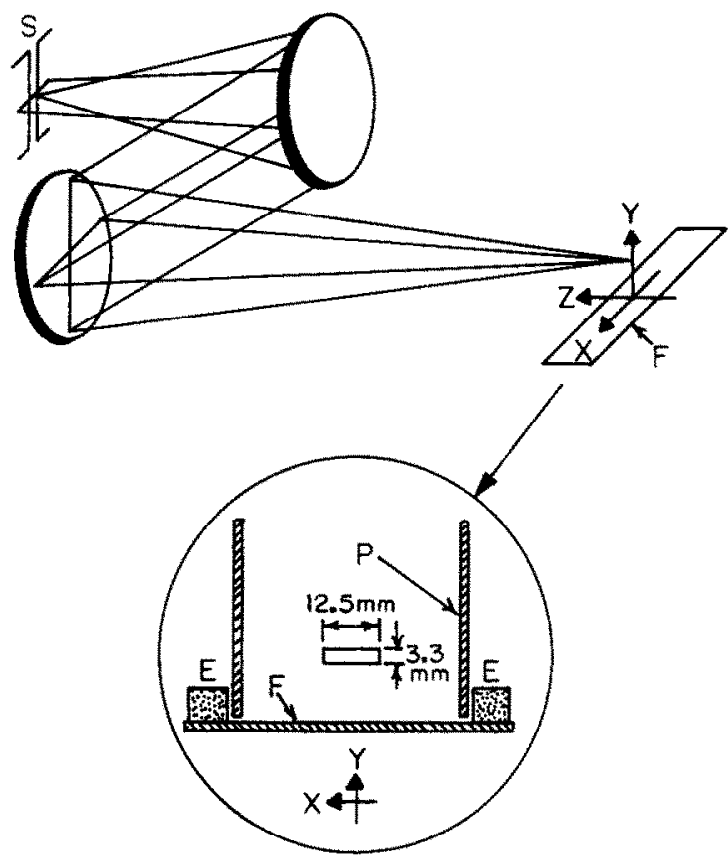

Fig. 2. Optical system used for spatially resolved photographic and photoelectric measurements. For photoelectric measurements, a $1 \mathrm{~mm}$ long entrance slit is used isolating a $3.3 \mathrm{~mm}$ high optical window in the plasma as shown in the inset. $F$, thin film on plastic substrate; $E$, graphite electrodes; $S$, entrance slit; $\mathbf{P}$, plastic shields.

integrated, spatially resolved photographic spectra were obtained on Kodak SA 1 plates which were processed according to manufacturer recommendations.

Discharge current waveforms were monitored with a Pearson Electronics wide-band current-tovoltage transformer (Model 1025). The voltage drop across the plasma was measured with a Tektronix Model P6015 capacitively-compensated high-voltage probe. All waveforms were obtained on a Nicolet Model 2090-III dual-channel digital storage oscilloscope which was equipped with a floppy-disk memory.

\subsection{Magnetic field measurements}

The transient magnetic field strength was measured with a probe coil consisting of 20 turns of AWG31 wire with a cross sectional area of $2.27 \mathrm{~cm}^{2}$. When a coil is placed in a time varying magnetic field such that the magnetic force lines are parallel to the coil axis, the induced voltage is given by Eqn (2).

$$
V=n A \mathrm{~d} B / \mathrm{d} t
$$

where $n$ in the number of turns in the probe coil, and $A$ is its cross-sectional area. The coil output signal was integrated with a passive $R C$ network giving a time varying voltage $V_{0}(t)$. The magnetic fieid strength then is found from this voltage and the values of $R$ and $C$.

$$
B=10^{8} V_{0} R C / n A
$$

where $B$ is in gauss, $V_{0}$ in volts, $R$ in ohms, $C$ in farads and $A$ in $\mathrm{cm}^{2}$.

This measurement technique was tested by placing the probe coil at the center of a long solenoid and comparing the measured field strength with the value calculated from Eqn (4).

$$
B=\mu_{0} I(t) n(\cos \alpha)
$$

where $I(t)$ is the current in the solenoid; $\alpha$ is the angle between the solenoid axis and a line connecting the center of the solenoid and a point on the circumference of either end, and $\mu_{0}$ is the magnetic permeability. Calculated and measured values differed by no more than $8 \%$.

\subsection{Materials and reagents}

Silver thin films were prepared on plastic substrates from $99.999 \%$ pure silver needles. Procedures are described in Ref. [15]. Aqueous test solutions were prepared from reagent-grade salts. Powder test materials were prepared from reagent-grade materials suspended in reagent-grade isopropyl alcohol. All experiments were conducted in a commercially prepared $40 \% \mathrm{O}_{2}-60 \%$ Ar gas mixture. 


\section{Results and Discussion}

\subsection{General plasma features}

Since the discharge current is used to generate the magnetic field, the field direction changes by $180^{\circ}$ for every half cycle of the oscillatory current. However, the electric field direction in the plasma also changes for every half cycle so the direction of the $E \times B$ drift motion is constant throughout the duration of the discharge. It is important to note that the external magnetic field strength is proportional to the instantaneous discharge current and it falls to zero at each zero-crossing of the current. Thus the magnetic coupling is relatively inefficient, and the plasma can expand freely for a short interval at the end of every half cycle.

The presence of the magnetic field has relatively little effect on the discharge current and plasma voltage drop [8]. A small frequency shift is observed which may be caused by a small change in magnetic permeability when the coil is on the discharge chamber relative to the aircore value.

Preliminary studies [8] showed that with the $E \times B$ drift motion directed vertically upward (away from the substrate surface) the plasma rapidly moves out of the observation zone and shows relatively little interaction with a sample deposited on the thin film surface. This configuration has relatively little analytical utility and will not be considered further. These same studies showed that with the $E \times B$ drift motion directed toward the substrate surface, the plasma appears to be confined to a relatively narrow region near the substrate; while without an external magnetic field, the plasma more-or-less uniformly fills the observation zone. These two cases will be compared in detail.

Figure 3 shows time integrated, spatially resolved spectra without an external field (a) and with the field present and the $E \times B$ drift motion directed toward the substrate surface (b). These spectra were obtained with the low-inductance discharge circuit using $6 \mathrm{kV}$ discharges. The two very strong lines are resonance lines from the $\mathrm{Ag}$ thin film. A number of other lines, which are marked in the figure, are from a $1.0 \mu \mathrm{g}$ sample of $5 \mu \mathrm{m}$ particles of Ni powder deposited on the thin film surface.

Several significant differences in these spectra are observed. First, the continuum background emission is much more localized with the magnetic field present, and the emission primarily is observed in a $15 \mathrm{~mm}$ wide region which begins at the substrate surface. In the no-field case, continuum radiation is more uniformly distributed through the $42 \mathrm{~mm}$ wide observation zone. With the field present, the continuum intensity is a maximum 1-2 mm from the substrate surface; while in the absence of the field, the maximum intensity is observed 6-7 $\mathrm{mm}$ from the surface.

The Ag neutral atom resonance lines are quite broad in both spectra but broadening near the substrate surface is considerably greater in the presence of the external magnetic field. In addition, relatively intense radiation from these lines is observed well below (about $10 \mathrm{~mm}$ ) the substrate surface. Without the magnetic field, this feature is completely absent. The neutral atom $\mathrm{Ni}$ lines from the powder sample show similar behavior.

The $1.6 \mathrm{~cm}$ wide $\mathrm{Ag}$ film is supported on a $4.5 \mathrm{~cm}$ wide plastic block. The presence of neutral atom radiation from the thin film and sample species below the surface of this support block can only occur if the magnetic field forces the plasma into this region. Thus it appears that the plasma is quite elastic, and the magnetic field may deform it more than compress it. That is, as the $E \times B$ motion of the plasma pushes it against the support block, it responds by expanding parallel to the surface of the block. If the plasma reaches the edge of the block, it will be forced downward by the drift motion. This suggests that the plasma must also be confined mechanically or magnetically in the direction normal to the drift motion if the drift motion is to result in significant plasma compression and subsequent heating.

An additional noteworthy feature observed in these time integrated, spatially resolved spectra is that ion lines from the Ag film material are restricted more to the region near the substrate surface than are the neutral atom lines with the magnetic field present. Without the field, ion and neutral atom lines show more similar distributions in the observation zone.

\subsection{Plasma expansion dynamics}

With or without the external magnetic field, photoelectric measurements show a progressive delay in the first appearance of radiation with increasing distance from the 


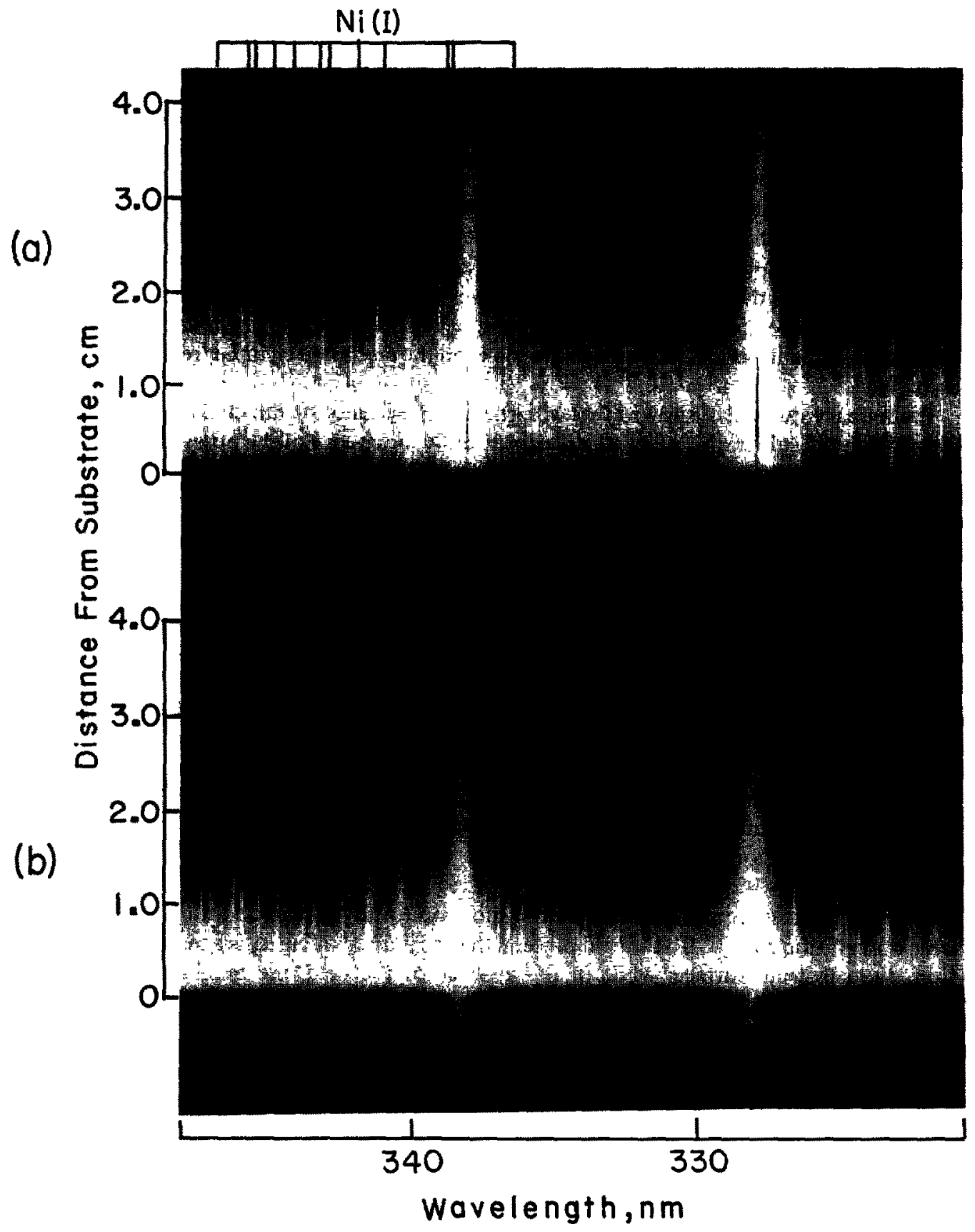

Fig. 3. Time integrated, spatially resolved photographic spectra of an electrically vaporized thin $\mathrm{Ag}$ film without (a) and with (b) an external magnetic field. For spectrum (b), the $E \times B$ drift motion of the plasma was directed toward the substrate surface which was located at the 0 -cm coordinate. The two very strong lines are the $\mathrm{Ag}$ neutral atom resonance lines at 328.1 and $338.3 \mathrm{~nm}$.

substrate to the observation zone. While the plasma boundary may be quite well defined, the image transfer system used here integrates radiation over a $3.3 \mathrm{~mm}$ vertical window. Thus, the sharpness of the leading edge of the radiation waveforms is determined in part by the time required for the leading edge of the plasma to move through the window.

Plasma propagation plots are shown in Fig. 4 for both the low-inductance and the highinductance discharges. These plots show the time of the first appearance of continuum radiation relative to the start of the discharge current as a function of the distance of the observation zone from the substrate surface. With the magnetic field present, the continuum radiation appears somewhat later in each observation zone. These plots indicate a more-or- 


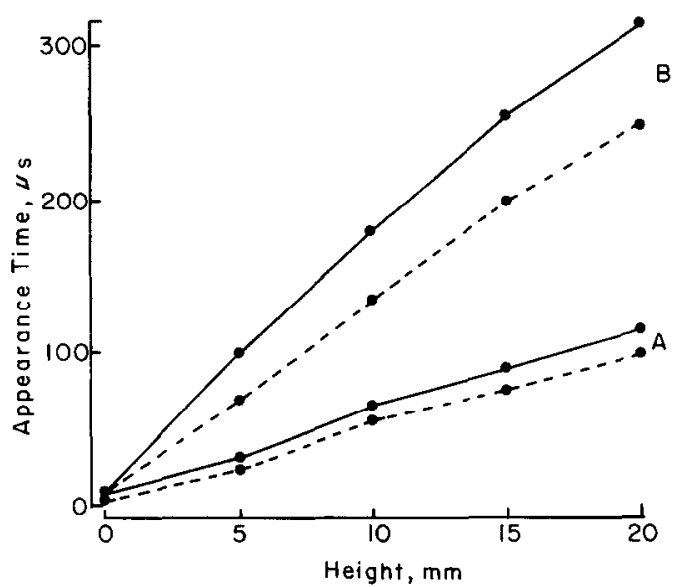

Fig. 4. Continuum background appearance time plots for low-inductance (A) and high-inductance (B) discharges with (solid lines) and without (broken lines) an external magnetic field.

less constant plasma expansion velocity in the region observed. Linear regression analysis of the plots from the low-inductance discharges gives expansion velocities of 180 and $200 \mathrm{~m} / \mathrm{s}$ with and without the magnetic field, respectively. Corresponding values for the highinductance discharges are 69 and $82 \mathrm{~m} / \mathrm{s}$, respectively. Thus, the presence of the magnetic field results in a $10-15 \%$ reduction in propagation velocity in this region of the plasma.

\subsection{Time and spatially resolved intensity measurements}

Fig. 5 shows continuum background radiation waveforms for the low-inductance $(105 \mu \mathrm{H})$ discharges both in the external magnetic field (solid lines) and with no magnetic field (broken

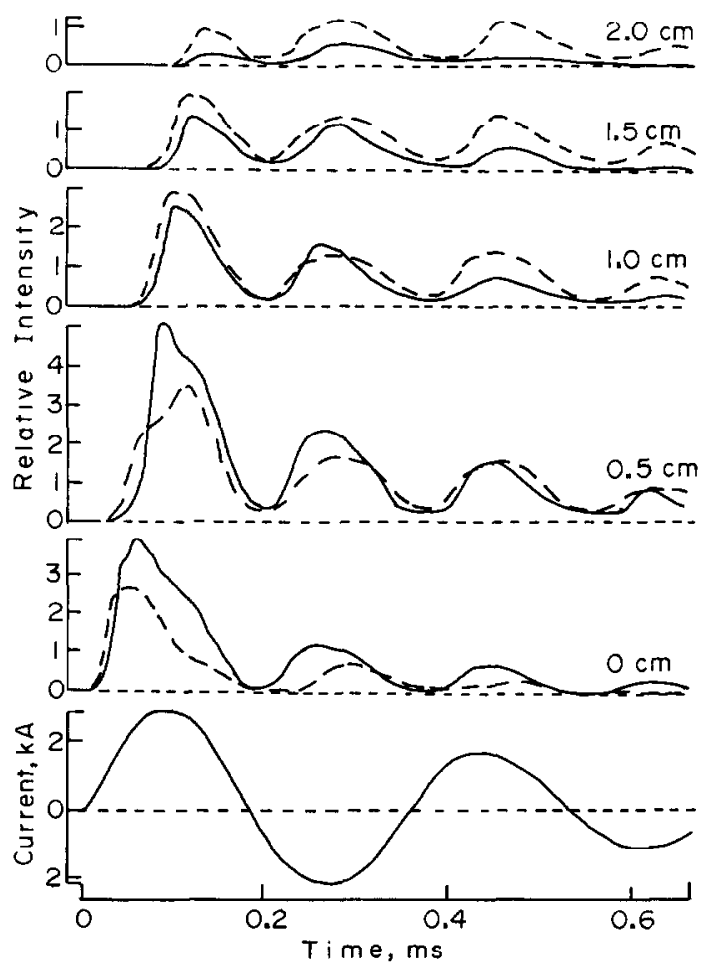

Fig. 5. Continuum background radiation profiles with (solid lines) and without (broken lines) an external magnetic field for various distances from the substrate surface using low-inductance discharges. The current waveform is provided as a time reference. 
lines). Measurements were obtained at five different distances from the substrate surface, and each waveform is an average from four experiments. The progressive delay in the first appearance of radiation with increasing distance from the substrate is clearly evident. At the substrate surface $(0 \mathrm{~cm})$ the continuum intensity is greater with the external magnetic field present for almost the entire duration of the discharge. The temporal variation in intensity also is greater with the field present. At $0.5 \mathrm{~cm}$ above the substrate, continuum intensity is greater than at $0 \mathrm{~cm}$ for both the field-on and the field-off cases. In part this is the result of reduced solid angle when viewing near the substrate surface. The intensities with and without the field are more similar at $0.5 \mathrm{~cm}$ than at $0 \mathrm{~cm}$. During the first two current half cycles, the intensity is greater with the field present. After the second half cycle, the intensity is slightly greater without the field. At $1.0 \mathrm{~cm}$ above the substrate, the intensity is greater without the field present for most of the discharge. This trend is even more apparent at 1.5 , and at $2.0 \mathrm{~cm}$, where the continuum intensity during most of the discharge is more than a factor-of-two lower in the external magnetic field.

Similar data are presented in Fig. 6 for the high-inductance discharge circuit. The trends observed in Fig. 5 for the low-inductance discharges also are observed in Fig. 6 but the differences between the field-off and the field-on cases are even more pronounced. At the substrate surface, the continuum intensity is about a factor of three greater with the field present. At $0.5 \mathrm{~cm}$, intensities are fairly comparable. At $2.0 \mathrm{~cm}$, the field-off intensity is nearly a factor of five greater than with the field present.

These data strongly suggest that the external magnetic field is confining the region of most intense continuum background to the region near the substrate surface. Differences in intensity with and without the field are greatest at the times of local current maxima and may be nearly negligible during periods of low discharge current. This is not surprising since the plasma current is used to generate the magnetic field, and thus the field strength is quite low during periods of low plasma current. This suggests that even more efficient control of the radiative properties of the plasma may be obtained with a unidirectional discharge current where zero crossings of the current are absent.

Figure 7 shows similar time and spatially resolved intensity profiles for the $\mathrm{Mn} 279.5 \mathrm{~nm}$ neutral atom line using low-inductance discharges. These waveforms were corrected for

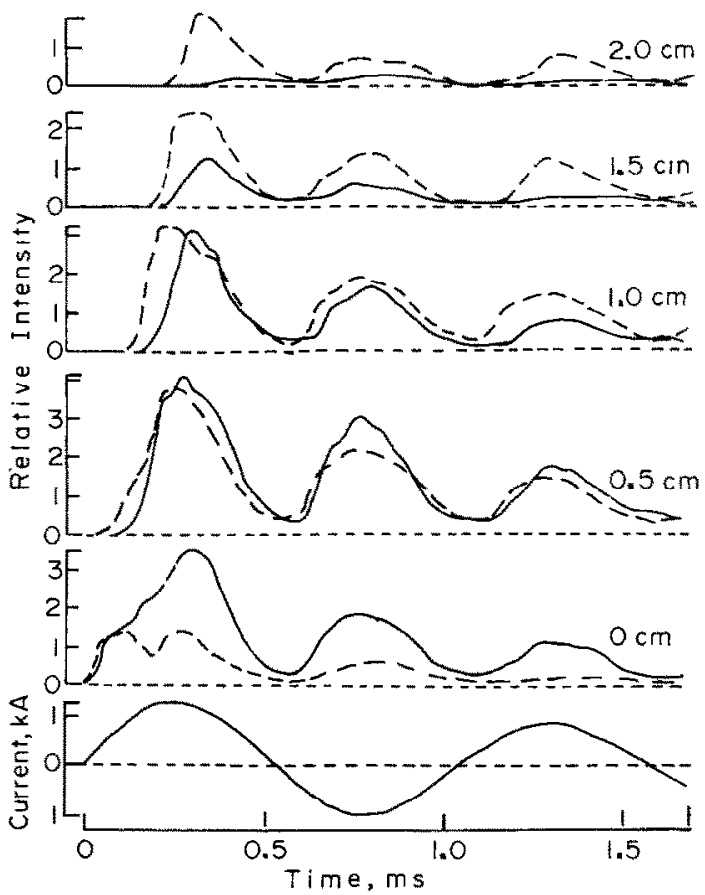

Fig. 6. Continuum background radiation profiles with (solid lines) and without (broken lines) an external magnetic field for various distances from the substrate surface using high-inductance discharges. The current waveform is provided as a time reference. 


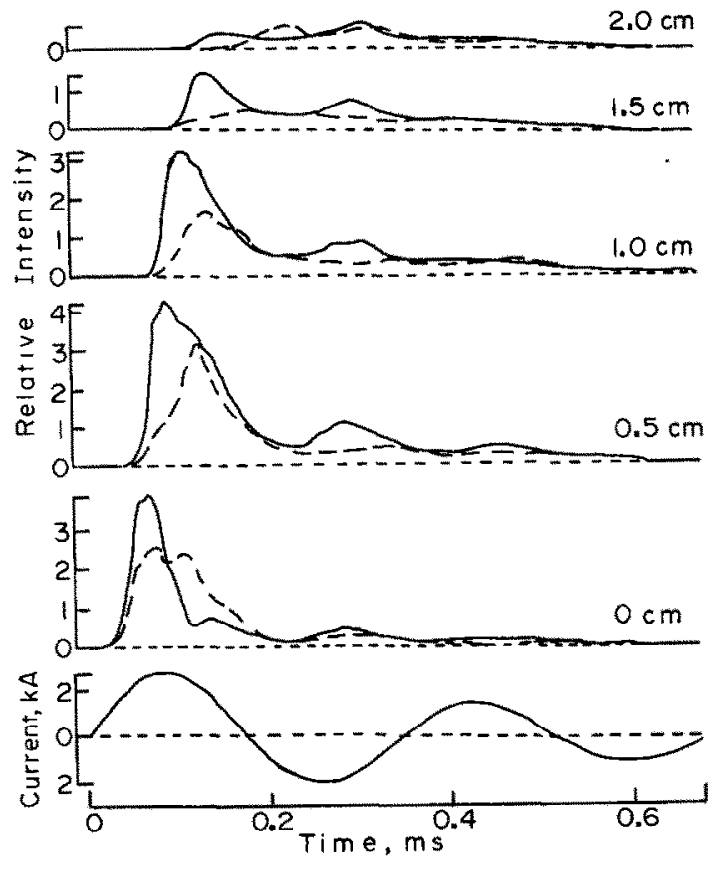

Fig. 7. Radiation profiles for the Mn $279.5 \mathrm{~nm}$ neutral atom line with (solid lines) and without (broken lines) an external magnetic field for various distances from the substrate surface using lowinductance discharges. The current waveform is provided as a time reference. A $0.5 \mu \mathrm{g}$ Mn powder sample was applied to each film.

continuum background, and each one is an average from four experiments. For each experiment, a $0.5 \mu \mathrm{g} \mathrm{Mn}$ powder sample was deposited on the substrate surface. The $\mathrm{Mn}$ particles used here passed through a $5 \mu \mathrm{m}$ pore diameter sieve, and thus the sample should vaporize completely before the end of the first current half cycle. At all distances from the substrate surface, radiation is first detected slightly earlier in the field-off case. However, the intensity increases much more rapidly and the intensity peak during the first current half cycle occurs earlier with the field present. In addition, except for the $2.0 \mathrm{~cm}$ case, the peak intensity is greater in the field-on case. Again, these features reflect the more rapid sample atomization when the $E \times B$ drift motion of the plasma keeps the current channel nearer to the substrate surface. Other studies have shown that sample vaporization may be an order-of-magnitude more rapid in this case.

After the first current half cycle, oscillations in the intensity profiles are greater with the field present, but the differences in intensity in the field-off and field-on cases are relatively smaller. After the second half cycle, intensities are about the same with or without the field at $1.0,1.5$ and $2.0 \mathrm{~cm}$ from the substrate surface.

Figure 8 shows similar profiles for the $\mathrm{Mn} 344,2 \mathrm{~nm}$ ion line. The Mn powder samples were the same as for Fig. 7. The differences between the field-on and the field-off cases are even more pronounced for the ion line. At the substrate surface, intensities integrated over the duration of the discharge are only slightly greater with the field present but local intensity maxima are shifted to earlier time in each current half cycle. This shift is also very obvious at other displacements from the substrate surface. Intensity oscillations are much greater in the field-on case, and in most cases, the intensity peaks are skewed with a fairly sharp rising edge and a more gradual falling edge. This is most apparent at 1.0 and $1.5 \mathrm{~cm}$ from the substrate surface. At displacements of $0.5-1.5 \mathrm{~cm}$, intensities integrated over the duration of the discharge are 2-3 times greater in the $E \times B$ plasma.

Similar trends are observed for the high-inductance discharges, but the differences in intensity between the field-on and field-off cases are less extreme. Significant skewing of the intensity peaks is observed with the field present. 


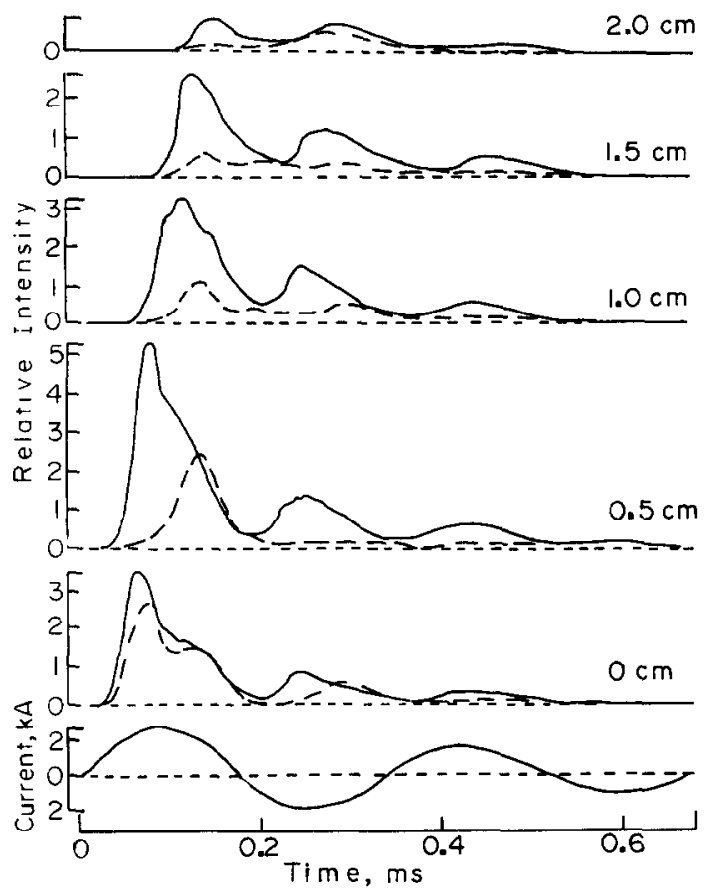

Fig. 8. Radiation profiles for the Mn $344.2 \mathrm{~nm}$ ion line with (solid lines) and without (broken lines) an external magnetic field for various distances from the substrate surface using low-inductance discharges. The current waveform is provided as a time reference. A $0.5 \mu \mathrm{g} \mathrm{Mn}$ powder sample was applied to each film.

\subsection{Analyte line-to-background intensity ratios}

The greater analyte line intensities coupled with lower continuum background intensities should result in very favorable line-to-background intensity ratios (L/B) in the $E \times B$ plasma for ion lines at displacements of $0.5 \mathrm{~cm}$ and greater from the substrate surface. Figure 9 shows L/B vs time plots obtained from the radiation profiles in Figs 5 and 8 for low-inductance discharges. Values of L/B were computed at $20 \mu$ s intervals directly from line and background waveforms stored in the digital storage oscilloscope.

At all displacements from the substrate surface and both with and without the external magnetic field, $L / B$ values vary significantly during the course of the discharge, and local maxima are observed after each zero crossing of the discharge current. These local maxima occur slightly later with increasing distance from the substrate surface.

At the substrate surface, $L / B$ values are greater without the magnetic field over nearly the entire course of the discharge. A peak value of over 30 is observed at the time of the first zero crossing of the discharge current. As the current begins to increase in the second half cycle, the $L / B$ value rapidly falls to a value of less than two. At all other displacements from the substrate surface, the L/B vs time plots have similar shapes with and without the magnetic field, but the plots are displaced to larger values when the plasma is in the magnetic field. The $\mathrm{L} / \mathrm{B}$ enhancement for the field-on case becomes progressively larger with increasing distance from the substrate.

Figure 10 shows similar $\mathrm{L} / \mathrm{B}$ vs time plots for the $\mathrm{Mn}$ ion line in the high inductance discharges. For these plots, values were obtained at $50 \mu$ s intervals. General features are similar to those in Fig. 9. However, at times of low discharge current, the continuum background intensity may be very low. This is particularly true at a displacement of $2.0 \mathrm{~cm}$ from the substrate. The result is a noisy $\mathrm{L} / \mathrm{B}$ plot with poorly defined values during intervals of low discharge current.

At the substrate surface, $\mathrm{L} / \mathrm{B}$ values are greater without the magnetic field except during short intervals after zero crossings of the discharge current. At other displacements from the substrate, L/B values are consistently larger with the field present. Very large values are observed at a displacement of $2.0 \mathrm{~cm}$ for the field-on case, but again, the data may not be 

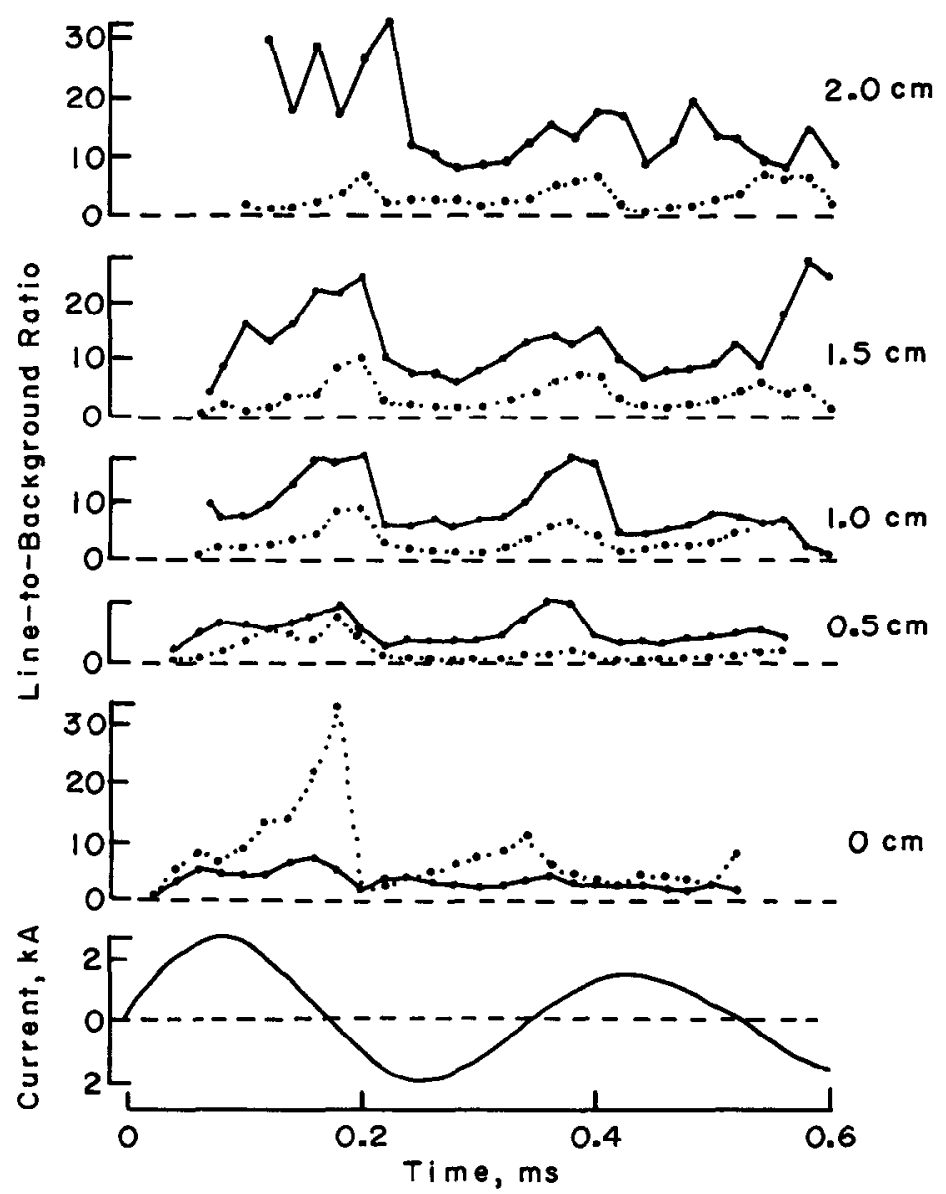

Fig. 9. Line-to-background ratios for the Mn $344.2 \mathrm{~nm}$ ion line with (solid lines) and without (dotted lines) an external magnetic field for various distances from the substrate surface using low-inductance discharges. The current waveform is provided as a time reference. A $0.5 \mu \mathrm{g} \mathrm{Mn}$ powder sample was applied to each film.

reliable because of the very low background intensity. In general, the plots for the field-on and field-off cases are not as parallel as for the low-inductance discharges. This is particularly obvious for the $1.5 \mathrm{~cm}$ plots where local maxima in the field-off plot occur at about the same times as local minima in the field-on plot.

\section{Conclusions}

It is apparent from the data presented in this report that relatively modest magnetic fields in an experiment of very simple design can have a very profound influence on the radiative properties of these transient, high-current capacitive discharge plasmas. The fact that the direction of plasma movement is normal to both the external magnetic field and the electric field in the plasma indicates that an $E \times B$ drift is the most reasonable explanation for the observed changes in plasma properties. There is relatively little literature on this type of plasma-magnetic field interaction at atmospheric pressure, and the microscopic equations for drift velocity may not be appropriate for a collision dominated plasma where ion and electron mean free paths are much less than their Larmor precession radii. It is probable that diffusion and ion mobility play significant roles in determining the macroscopic plasma trajectory.

It also appears that the plasma may not be confined significantly by the magnetic field, and additional magnetic or mechanical confinement normal to the direction of the plasma drift may be required to increase charge density and gas kinetic temperature in the plasma. However, improved interaction of the plasma with a sample on the substrate surface appears 


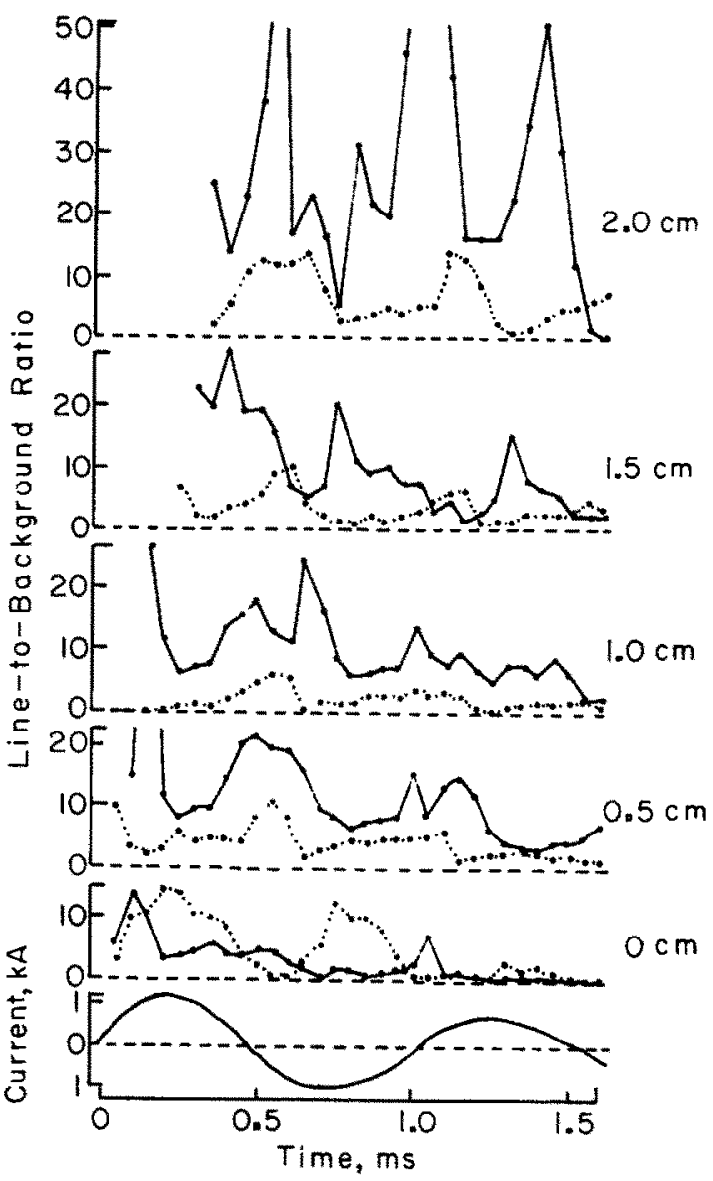

Fig. 10. Line-to-background ratios for the $\mathrm{Mn} 344.2 \mathrm{~nm}$ ion line with (solid lines) and without (dotted lines) an external magnetic field for various distances from the substrate surface using highinductance discharges. The current waveform is provided as a time reference. A $0.5 \mu \mathrm{g} \mathrm{Mn}$ powder sample was applied to each film.

to result in much more rapid sample atomization. This should be of considerable analytical utility, particularly for powder samples containing relatively large particles.

The increased L/B values observed with the magnetic field for displacements $1 \mathrm{~cm}$ or more from the substrate surface indicate that the plasma may become more spatially heterogeneous in the magnetic field, and the region of most intense continuum background is separated from regions of intense analyte line emission. Optical masking of the region near the substrate surface then should provide a simple method for reducing detection limits. Preliminary data have confirmed this. However, for single-channel measurements using a prerecorded background correction, the reductions may not be as significant as is indicated in Figs 9 and 10. This is because of poorer shot-to-shot reproducibility of the continuum background intensity when only a portion of the plasma is observed. This results in a smaller increase in signal-to-noise ratio. Larger increases in signal-to-noise ratio should be realized if dual-channel measurements are made. 